\title{
Prácticas relacionadas con la salud en estudiantes de primer ingreso de la Licenciatura en Trabajo Social de la \\ Universidad de Sonora
}

César Walterio Varela, Karina Manríquez, Karen Amaya,

David Ocaño y Jéssica Peñúñuri

Eduardo A. Vizer* Helenice Carvalho**

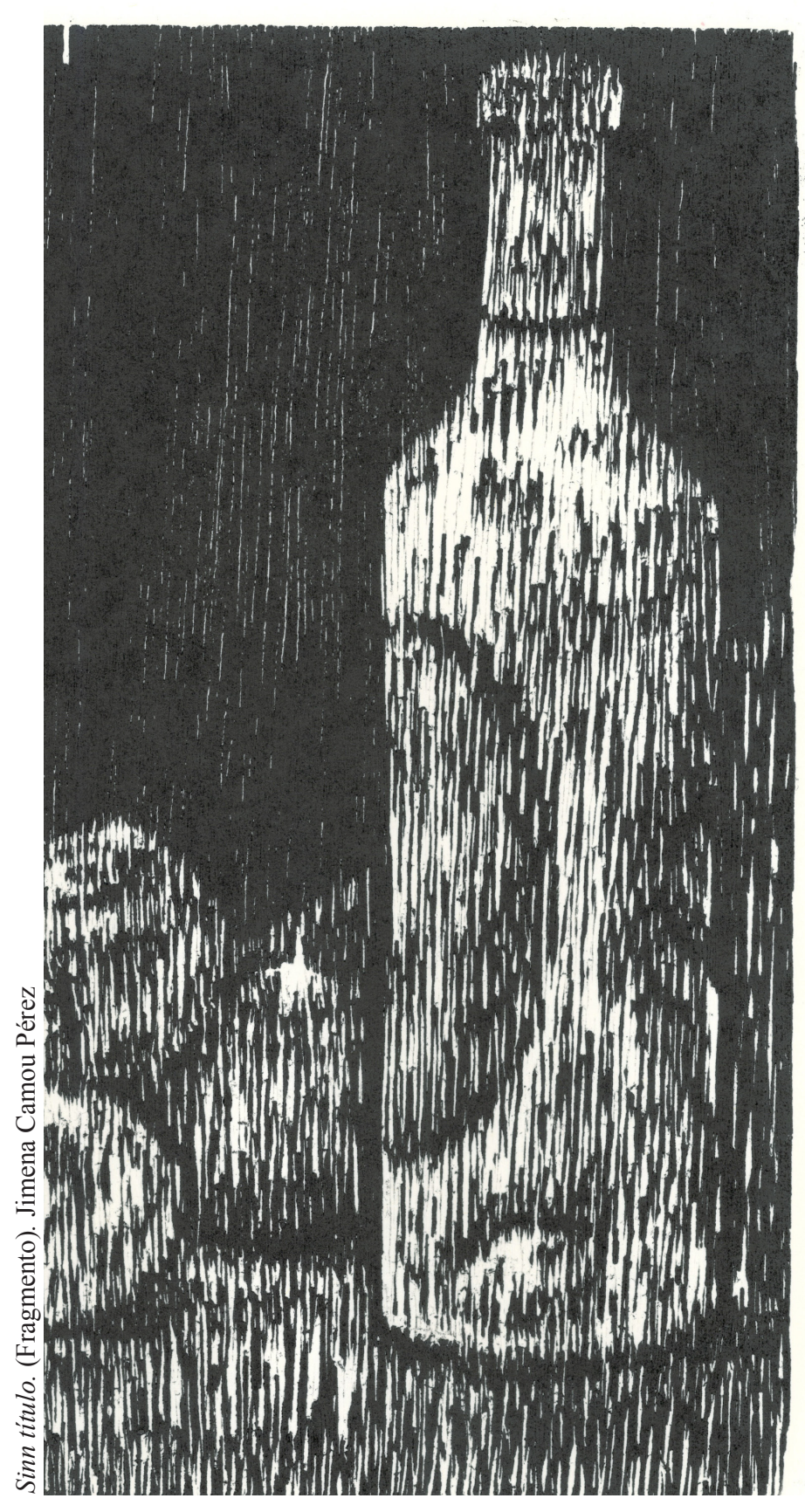

\section{Introducción}

Investigaciones de carácter diagnóstico en aspectos relacionados con la salud en ésta y en otras universidades del país (Varela, 2007; Varela y cols., 2007; López y González, 2002) arrojan resultados consistentes sobre la situación en la que se encuentran un buen número de estudiantes de nivel superior.

Esto ha tenido un profundo efecto en la responsabilidad de la institución educativa: si bien es incuestionable el papel tradicional de esforzarse en ofrecer las mejores oportunidades en la formación académica y profesional de los estudiantes (actividad primordial o sustantiva), aparece un segundo objetivo que se refiere a la preocupación por parte de la institución educativa de informarse sobre condiciones biológicas, psicológicas y sociales que pudieran configurarse como factores ya sea de protección o de riesgo, y que en ese sentido, favorezcan o impidan el desarrollo humano en general del alumno y a partir de esto, el diseño e implementación de programas de evaluación e intervención acordes a la problemática de la población estudiantil.

En ese sentido, la disposición de información en estas dimensiones (aspectos de integridad, biológica, psicológica y social) ha permitido dilucidar sobre la problemática presente y potencial de los estudiantes, y cómo esta situación condiciona la necesidad de una nueva funcionalidad en la organización material y administrativa de la universidad para responder a estas demandas en el ámbito de la salud en un amplio sentido (López y González, 2005, Figueroa, 2005).

Los estudios sobre el estatus de salud en estudiantes de nivel medio y superior ya sea como condición actual o como evento probabilístico a partir de prácticas de vida y/o condiciones generales que los rodean se han multiplicado en las últimas fechas. El espectro de alteraciones en la salud y/o condiciones con ella relacionada es muy amplio, y generalmente se organizan en rubros relacionados con el nivel de expresión de las mismas: alteraciones relacionadas con el sustrato 
biológico y/o salud física, alteraciones en la esfera de lo psicológico o conductual y condiciones sociales (incluyendo aspectos del ambiente físico) que afectan el desarrollo de la salud.

Los antecedentes de este tipo de estudios se ubican en la relativamente nueva tradición de la descripción epidemiológica de aspectos relacionados con la salud en la población tanto adolescente en general, como los estudiantes universitarios. Los adolescentes aumentaron en un $22 \%$ en el siglo XX; es también un grupo, que si bien, es relativamente sano, es decir, utiliza menos servicios de salud y en él se presentan menos defunciones. Por otro lado, la adolescencia es la época del inicio a factores de riesgo como el sexo no seguro, el consumo de tabaco, alcohol y drogas ilícitas, prácticas inadecuadas de alimentación, sedentarismo, y con esto, la aparición de las condiciones que hacen probable las enfermedades crónico degenerativas propias del adulto. Sus prácticas de vida, así como sus antecedentes familiares (entre otros factores), los colocan en condiciones de riesgo en un mediano o largo plazo.

La preocupación por los patrones de comportamiento relacionados con la salud en estos grupos etarios es fácilmente justificada; por un lado, nos encontramos en una etapa de la vida de la persona en donde no se favorece el análisis crítico de las condiciones generales de vida y con esto la elección de comportamientos generalmente elegidos por los adolescentes, además del intenso ímpetu que históricamente se le ha reconocido a la adolescencia (Steinberg, 2004). Por otro lado, las estadísticas nos señalan la alta incidencia de este grupo etario en situaciones que los acerca al riesgo, a la afectación, o a la misma muerte (Blum \& Ireland, 2004; Blum \& Nelson-MMari, 2004; Ruangkanchanasetr, Plitponkarnpim, Hetrakul \& Kongsakon, 2005; Willis, 2004). Es de esperar que el desarrollo de programas de intervención en el área de la salud derive de las problemáticas específicas reportadas en los diversos grupos sociales.

\section{Objetivo general}

Obtener información diagnóstica sobre factores relacionados con el estatus de salud de los estudiantes del primer año de la Licenciatura en Trabajo Social de la Universidad de Sonora (Campus Centro).

\section{Metodología y técnicas \\ Participantes}

Alrededor de 89 estudiantes del primer año (ciclo 2009-2- 2010-1) de la Licenciatura en Trabajo Social de la Universidad de Sonora, Campus Hermosillo.

\section{Procedimiento}

La fase de levantamiento de datos se realizó en el ciclo escolar 2009-1 y 2009-2, se pasó a los salones previa plática solicitando el apoyo de autoridades y maestros.

Los instrumentos fueron aplicados en el aula y son de carácter anónimo y voluntario.

\section{Instrumentos}

Se utilizaron varios cuestionarios y escalas. Para el caso del fumar, se seleccionaron algunas de las escalas de la Encuesta Mundial de Tabaco para la Juventud (EMTJ), para la ingesta de alcohol, se adaptaron la mayoría de las escalas de tabaquismo, adaptándolas al consumo de alcohol; un cuestionario sobre sexualidad, una serie de reactivos sobre prácticas de deportes y actividades recreativas y trabajo y preguntas sobre ingesta de drogas ilícitas.

\section{Resultados}

Con respecto a las prácticas de fumar, y tomar bebidas alcohólicas, del total de los estudiantes (89), el $9 \%$ (8) reporta ser un fumador habitual, y el 31\% (27) ser tomador habitual; con respecto a la edad de inicio $\mathrm{y}$, para el caso de fumar, la mayoría reporta haberse iniciado entre los 14 y los 17 años (5, representando un $63 \%$ ) y 2 alumnos (25\%) de los 18 a los 21 años. Con respecto a la ingesta de bebidas alcohólicas de una manera habitual, 16 alumnos (18\%) y $9(10 \%)$ entre los 18 y los 21 años; 2 (2\%) de los alumnos reportan fumar entre 6 a 19 días, muy parecido (3, el 3\%) de 20 a 29 días; solo el 5\% (4 alumnos) reporta haber fumando de 1 a 5 cigarros por día. Casi la totalidad de los alumnos (98\%) reporta que fumar tabaco es dañino para la salud; en relación a la exposición al humo de segunda mano, el $28 \%$ ( 25 alumnos) se expone de 1 a 4 días a la semana, el $14 \%$ de 5 a 7 días, mientras que la exposición al humo estando fuera de su casa se incrementa enormemente: el $46 \%$ (41 alumnos) de 1 a 4 días, y el 30\% (27 alumnos) de 5 a 7 días. 
Solo el 1\% de los estudiantes que fuma se levanta algunas veces con las ganas de encender un cigarro. Del total de alumnos, solo el 12\% (11 estudiantes) reporta que probablemente lo seguirá haciendo en los próximos 12 meses y cero que lo hará con seguridad; presentándose los mismos valores $(11 \%$ y $0 \%)$ a una perspectiva de 5 años; con respecto a la práctica de ingerir bebidas alcohólicas y en un lapso de 12 meses, un 46\% (41 alumnos) reporta que probablemente si lo seguirá haciendo, y un 6\% (5 alumnos) que definitivamente si lo estará haciendo, y para una perspectiva a 5 años, un $43 \%$ (38 alumnos) reporta que muy probablemente lo hará y un 6\% (5 alumnos), definitivamente lo estará haciendo.

La mayoría de los estudiantes reporta que fumar $(96 \%)$ y tomar (98\%) son dañinos para la salud; sin embargo, su exposición al humo de segunda mano es alta, principalmente fuera de su casa (un $28 \%$ de uno a cuatro días y el 14\% entre 5 a 7 días dentro de su casa, mientras que fuera de su casa se expone un $46 \%$ de los alumnos (41) de uno a cuatro días y hasta el 30\% de 5 a
7 días; un 65\% de los alumnos (57) reporta que algunos de sus amigos fuman, el 18\% (16) que la mayoría, y un $1 \%$ que todos sus amigos lo hacen. Con respecto a la ingesta de alcohol, 12 alumnos (13\%) reportan que todos sus amigos lo hacen, el 40\% (34) reporta que la mayoría de ellos, y el $40 \%$ (36) reporta que algunos lo hacen; $7(8 \%)$ alumnos reportan que ambos padres fuman, 16 $(18 \%)$ que solo lo hace su padre, y $8(9 \%)$ que solo lo hace su madre; con respecto a la ingesta de alcohol, el $19 \%$ (17) de los padres (ambos) toman, el 20\% (18) para el caso del padre y el 33\% (29) en el caso de la madre.

El 100\% (8) de los estudiantes que fuman, creen que podrían dejar de hacerlo si quisieran, así como un $60 \%$ (18) de los que ingieren alcohol habitualmente, creen que podrían dejarlo por la misma razón. Un $62 \%$ de los estudiantes que fuman y/o toman (30\%), reporta querer dejar de hacerlo, o de controlarlo, y hasta un 75\% estaría dispuesto a recibir apoyo de especialistas en el caso del fumar y $60 \%$ del tomar (Tabla 1 ).

Tabla 1. Principales datos sobre fumar e ingerir bebidas alcohólicas.

\begin{tabular}{|c|c|c|c|}
\hline & \multicolumn{2}{|r|}{ Fumar-Tabaco } & Tomar-Alcohol \\
\hline - Consumidor habitual & & $89 / \%$ & $27 \quad 31 \%$ \\
\hline $\begin{array}{l}\text { - El tabaco-alcohol es } \\
\text { dañino para la salud }\end{array}$ & $\begin{array}{l}\text { Probable } \\
2 \quad 2 \%\end{array}$ & $\begin{array}{l}\text { Definitivo sí } \\
8494 \%\end{array}$ & $\begin{array}{ll}\text { Probable sí } & \text { Definitivo sí } \\
9 \quad 10 \% & 7888 \% \\
\end{array}$ \\
\hline $\begin{array}{l}\text { - Si quisieras, podrías } \\
\text { dejar... }\end{array}$ & 8 & $9 \%$ & $16 \quad 60 \%$ \\
\hline $\begin{array}{l}\text { - Quiero dejar, o limitar mi } \\
\text { manera de ... }\end{array}$ & 5 & $62 / \%$ & $8 \quad 30 \%$ \\
\hline $\begin{array}{l}\text { - ¿Recibirías ayuda para } \\
\text { dejar? }\end{array}$ & 6 & $75 \%$ & $16 \quad 60 \%$ \\
\hline $\begin{array}{l}\text { - ¿Es dañino el humo de } \\
\text { segunda mano? }\end{array}$ & $\begin{array}{l}\text { Probable } \\
6 \quad 7 \%\end{array}$ & $\begin{array}{l}\text { Definitivo sí } \\
80 \quad 90 \%\end{array}$ & \\
\hline $\begin{array}{l}\text { Fumador pasivo } \\
\text { Dentro de casa } \\
\text { Fuera de casa }\end{array}$ & $\begin{array}{l}1 \text { a } 4 \text { días } \\
25 \quad 28 \% \\
41 \quad 46 \%\end{array}$ & $\begin{array}{l}5 \text { a7 días } \\
13 \quad 14 \% \\
27 \quad 30 \%\end{array}$ & \\
\hline $\begin{array}{l}\text {-Durante el último mes } \\
\text { ¿Cuántos días? } \\
\text { ¿Cuántos cigarros? }\end{array}$ & $\begin{array}{l}6 \text { a } 19 \\
2 \quad 2 \% \\
1 \text { a } 5 \\
45 \% \\
\end{array}$ & $\begin{array}{ccc}20 \text { a } 29 & \text { Todos } \\
3 \quad 3 \% & 0 \\
6 \text { a } 20 & \text { Más de } 20 \\
0 & 0 \\
\end{array}$ & 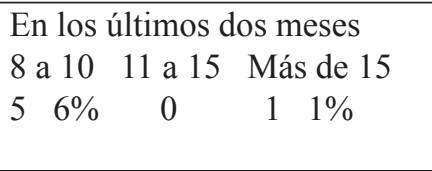 \\
\hline $\begin{array}{r}\text { - Perspectiva temporal } \\
\text { A un año estaré } \\
\text { A } 5 \text { años estaré }\end{array}$ & 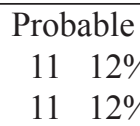 & $\begin{array}{lc}\text { sí } & \text { Definitivo sí } \\
0 \\
0\end{array}$ & 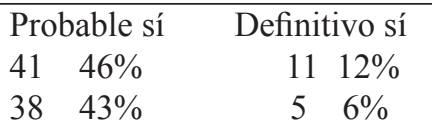 \\
\hline - Mis papás & $\begin{array}{l}\text { Los } 2 \\
78 \%\end{array}$ & $\begin{array}{cl}\text { Papá } & \text { Mamá } \\
2326 \% & 89 \%\end{array}$ & 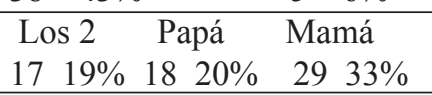 \\
\hline - Mis amigos & $\begin{array}{l}\text { Algunos } \\
5765 \% \\
\end{array}$ & $\begin{array}{ccc}\text { Mayoría } & \text { Todos } \\
6 \quad 18 \% & 11 \% \\
\end{array}$ & 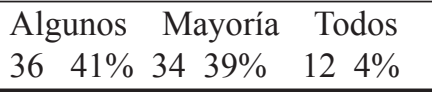 \\
\hline
\end{tabular}


En relación a los padecimientos diversos hacia el interior de la familia, y con respecto a la práctica de fumar, $9 \%$ de los estudiantes reporta ser fumador habitual, así como referir el $27 \%$ en su padre, el $16 \%$ en su madre y el $21 \%$ en sus hermanos; con respecto a la ingesta de alcohol, el $27 \%$ se define como tomador habitual, el $60 \%$ en su padre, el $16 \%$ en su madre y el $25 \%$ en sus hermanos. Para el caso del uso de drogas ilícitas, ninguno de ellos reporta haber tenido experiencias y/o seguir usando, lo mismo para el caso del padre o de la madre, solo un seis \% en sus hermanos. Para el caso de la diabetes, se presenta un $1 \%$ tanto en los alumnos como en sus hermanos, un $9 \%$ en el caso del padre y un $15 \%$ en el caso de la madre. Para la presión arterial alta (o problemas de presión en general) los estudiantes reportan un $2 \%$ en ellos, un $0 \%$ en sus hermanos y un $15 \%$ y un $24 \%$ en el caso del padre y de la madre. En el caso de las afecciones del corazón, tenemos un $1 \%$ en los estudiantes, y un $7 \%$ y un $6 \%$ padre y madre, respectivamente. En el caso de los episodios depresivos, encontramos un $36 \%$ en los alumnos, un $1 \%$ en los hermanos y un 4 y $10 \%$ para padre y madre; en el caso del cáncer, un $2 \%$ en el caso de el padre y los hermanos y un $1 \%$ en el caso de la madre, no se presentan casos en los estudiantes; por último, para la obesidad (problema de niveles epidémicos en el país, sobre todo en el estado de Sonora, y con un gran peso en términos de afectaciones en múltiples sistemas del organismo), se presenta un $11 \%$ en los estudiantes, un $7 \%$ en sus hermanos, y un $15 \%$ en el caso de el padre y un $12 \%$ en la madre (Tabla 2).

Tabla 2. Porcentaje de aparición de los diversos padecimientos hacia el interior de la familia reportado por el estudiante.

\begin{tabular}{|l|c|c|c|c|}
\hline Padecimiento & Estudiante & Padre & Madre & Hermanos \\
\hline Alcohol & 27 & 60 & 16 & 25 \\
Tabaco & 9 & 27 & 16 & 21 \\
Drogas Ilícitas & 0 & 0 & 0 & 6 \\
Diabetes & 1 & 9 & 15 & 1 \\
Presión Alta & 2 & 15 & 24 & 0 \\
Corazón & 3 & 7 & 6 & 4 \\
Depresión & 36 & 4 & 10 & 1 \\
Cáncer & 0 & 2 & 1 & 2 \\
Obesidad & 11 & 15 & 12 & 7 \\
\hline
\end{tabular}

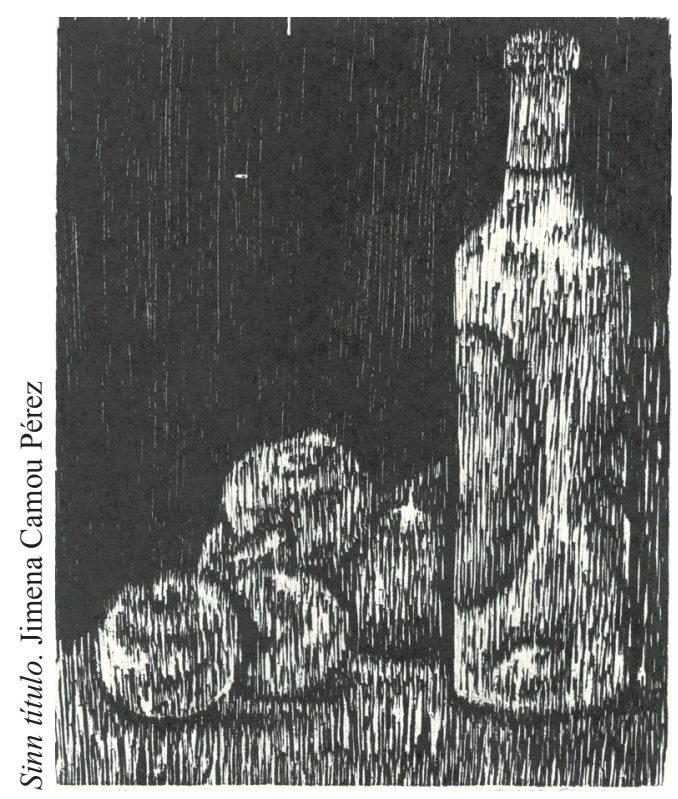

En el caso de la escala de Ideación e intento suicida, los estudiantes reportan en $37 \%$ el haber tenido la sensación de que no vale la pena vivir, o haber experimentado serias depresiones, un 7\% el haber experimentado suficiente tensión como para intentar suicidarse y un $2 \%$ con la concreción del intento suicida.

En relación a la sexualidad, la mayoría (81\%) reporta no acudir al servicio médico a realizarse revisiones relacionadas con su sexualidad, el $8 \%$ lo hace a la larga y solo el $9 \%$ lo hace regularmente; alrededor del $43 \%$ reporta la práctica sexual, de éstas, el $47 \%$ son planeadas, el 26\% la mayoría de las veces, el 9\% la minoría de ellas y el 18\% reporta no planearlas; el 76\% reporta haber usado preservativo en la primera ocasión y usar el condón (68\%) entre siempre y la mayoría de las veces, los estudiante reportan usar el ritmo $(18 \%)$ y el coitus interruptus $(22 \%)$ entre siempre o la mayoría de las veces; se reporta no haber padecido infecciones de transmisión sexual.

En relación a la práctica de deportes, el 33\% reporta incluirlas en sus actividades diarias, así como al realizar otras actividades recreativas (12\%), el 25\% reporta trabajar además de estudiar (Tabla 3).

En relación a la ingesta de drogas ilícitas, los valores son en general bajos: el 7\% reporta haber tenido experiencias anteriormente, pero ya no hacerlo, lo mismo para el caso de la cocaína en un $3 \%$ y de las anfetaminas en un $1 \%$, de $4 \%$ en el caso de los tranquilizantes y 2 estudiantes utilizar esta droga varias veces al año. 


\section{渵 Sonta}

Tabla 3. Principales datos sobre sexualidad, deportes y actividades recreativas.

\begin{tabular}{|c|c|c|c|c|}
\hline & Regular & A la larga & No & \\
\hline - ¿Acudes al médico a revisiones? & $9 \%$ & $8 \%$ & $81 \%$ & \\
\hline - ¿Tienes relaciones sexuales? & Sí: 43\% & & & \\
\hline $\begin{array}{l}- \text { ¿Son tus contactos sexuales } \\
\text { planeados? }\end{array}$ & $\begin{array}{l}\text { Sí plan } \\
47 \%\end{array}$ & $\begin{array}{l}\text { Mayoría } \\
26 \%\end{array}$ & $\begin{array}{l}\text { Minoría } \\
9 \%\end{array}$ & $\begin{array}{l}\text { No plan } \\
18 \%\end{array}$ \\
\hline $\begin{array}{l}\text { - ¿Usaste preservativo en tu primer } \\
\text { contacto? } \\
\text { - Preservativo más usado } \\
\text { - Condón } \\
\text { - El ritmo } \\
\text { - Coitus interruptus }\end{array}$ & $\begin{array}{l}\text { Sí: } 76 \% \\
\begin{array}{lc}68 \% & \text { (entre siempre } \\
18 \% & \text { y la mayoría } \\
22 \% & \text { de las veces) }\end{array}\end{array}$ & & & \\
\hline $\begin{array}{l}\text { - Infecciones de transmisión sexual? } \\
\text { - ¿Practicas deportes? } \\
\text { - ¿Practicas actividades recreativas? } \\
\text { - ¿Trabajas? }\end{array}$ & $\begin{array}{l}\text { Cero infecciones } \\
58 \% \\
17 \% \\
29 \%\end{array}$ & & & \\
\hline
\end{tabular}

\section{Conclusiones}

Los resultados del trabajo son importantes, ya que si bien, no se traducen directamente en condiciones de afectación biológica, psicológica o social para los estudiantes, sí representan una valiosa información sobre el posible curso de acontecimientos que podría terminar con problemas en los tres niveles referidos. Inicialmente podríamos mencionar los problemas relacionados con la conducta de fumar e ingerir bebidas alcohólicas, que en general coinciden con estudios realizados en la misma Universidad (Varela, 204), en el país o en los países industrializados, aunque ligeramente bajos en comparación a otras escuelas de la División de Ciencias Sociales de la misma Universidad. Se subraya la expectativa a fumar y tomar en uno a cinco años, así como la familiaridad y por lo tanto aceptación y práctica de estas conductas tanto en los padres como en los amigos, así como en la exposición al humo de segunda mano en los estudiantes tanto en su casa, como fuera de ella (permisibilidad). Estos datos sugieren la necesidad de desarrollar esfuerzos para limitar esas prácticas vistas como algo natural e indiferente a los no fumadores (y hacia ellos mismos), e instaurar lugares libres de dichas prácticas. Por otro lado, es interesante el gran número de alumnos que quisieran dejar o controlar sus prácticas en relación al consumo de tabaco y alcohol, entre otros, dato a considerar por las instancias relacionadas con la salud en la universidad, y que la obliga a desarrollar mecanismos a nivel estructural y administrativo para ofrecer salidas a estas problemáticas con graves consecuencias a corto, mediano y largo plazo, pensando en la reactividad del estudiante a los requerimientos que la enseñanza le impone, y a mediano y largo plazo, en la gran serie de problemas que estas prácticas acarrean.

En un estudio previo sobre estas prácticas en estudiantes de todo el Campus Centro de la misma universidad (Varela y cols., 2007), los valores de exposición al humo de segunda mano, el deseo de querer controlar y/o dejar esas prácticas a partir de su propia decisión, la edad de inicio como fumadores o bebedores de alcohol, las expectativas a seguir fumando y/o tomando a uno o cinco años, y las prácticas en sus padres y amigos son muy similares, lo cual nos habla de la tendencia en esta problemática en la población universitaria

En relación a los padecimientos diversos, quisiéramos mencionar los casos de los reportes sobre depresión y obesidad, mismos que consideramos muy importantes, el primero, por las implicaciones sobre el estatus actual del desenvolvimiento del alumno y el segundo por implicaciones a mediano y largo plazo, ya documentadas en la literatura internacional, y de proporciones epidémicas en el país y en particular en nuestro estado. Interesante mencionar las cifras en los padecimientos crónico degenerativos (diabetes, presión arterial, 
alteraciones en el corazón y cáncer), ya que los valores de los padres, reflejan la tendencia (vía genética, aunada a variables contextuales y concretizada en prácticas de los hijos), que se reflejará sus hijos en la edad adulta y la vejez. Y por supuesto, no olvidar los datos sobre ideación e intento suicida, evidentemente preocupante que ameritan la intervención de tipo profesional a corto plazo.

En relación a la sexualidad, subrayar la importancia que tienen estas prácticas, y las implicaciones que sobre su salud, y la de sus parejas tienen al alejarse del cuidado esencial (escaso número de revisiones médicas, inicio temprano, uso de métodos poco efectivos como el coitus interruptus o el ritmo, etcétera).

Consideramos importante la realización de este tipo de estudios, ya que deberían de reflejarse en mejoras en las condiciones de alumnos (López y González, 2005; Varela, 2007; Adelman y Taylor, 2000) y así, potencializar sus logros académicos, alejándolos de prácticas relacionadas con la afectación fisiológica, psicológica y social que impacten su desarrollo tanto en su tránsito en su formación profesional, como en su vida dentro de la sociedad.

\section{Bibliografía}

Adelman, H. S. \& Taylor, L. (2000). Shaping the future of mental health in school. Psychology in the Schools. 37, 49-60.

Blum, R. W. \& Ireland M. (2004). Reducing risk, increasing protective factors: Findings from the caribbean youth health survey. Journal of Adolescent Health. 35, 493-500.

Blum, R. W. \& Nelson-Mmari, K. (2004). The health of young people in a global context. Journal of Adolescent Health. 35, 402-418.

Figueroa, V. D. (2005). Salud e higiene universitaria: Objetivo estratégico para los centros de educación superior. Típica. Boletín Electrónico de Salud Escolar. 1, No. 1.

López B. J. y González de C. M. (2002). Examen Médico Automatizado. México: UNAM.

López B. J. y González de C. M. (2005). Modelo Universitario de salud integral en la Universidad Nacional Autónoma de México. Revista de la Facultad de Medicina. UNAM: 48(6): 224-231.
Ruangkanchanasetr, S., Plitponkarnpim, A., Hetrakul, P. \& Kongsakon, R. (2005). Youth risk behavior survey: Bangkok, Thailand. Journal of Adolescent Health. 36, 227- 235.

Steinberg, L. (2001). Theories of adolescent development. International Encyclopedia of the Social \& Behavioral Sciences. pp. 102-105. Elsevier Science. Ltd.

Varela, R. C. (2007). La Universidad y el estado de salud del estudiante universitario. En González Lomelí, D. y Maytorena, M. Estudios empíricos en educación superior. México: UNISON.

Varela, R., Reyes, L. U., Cabrera, R. , Vejar, V. y Ruiz, R. (2007). Estilos de aprendizaje, ingesta de tabaco, alcohol y drogas ilícitas y rendimiento académico. Revista Mexicana de Psicología. XV Congreso Mexicano de Psicología. pp. 190-191.

Willis, T. (2001). Adolescents health and health behaviors. International Encyclopedia of the Social \& Behavioral Sciences. pp. 105-112. Elsevier Science. Ltd. 